\title{
Synovitis, Acne, Pustulosis, Hyperostosis, and Osteitis Syndrome
}

National Cancer Institute

\section{Source}

National Cancer Institute. Synovitis, Acne, Pustulosis, Hyperostosis, and Osteitis

Syndrome. NCl Thesaurus. Code C119049.

A syndrome characterized by synovitis, acne, pustulosis, hyperostosis, and non-infectious osteomyelitis. Includes a spectrum of aseptic neutrophilic dermatoses associated with aseptic osteoarticular lesions. 